\title{
L'arbuste Calotropis procera, un épisode de son histoire dans le bassin du lac Tchad
}

Calotropis procera, an episod of its story in the Lake Chad Basin

\section{Christian Seignobos}

\section{CpenEdition}

\section{Journals}

Édition électronique

URL : http://journals.openedition.org/ethnoecologie/2462

DOI : $10.4000 /$ ethnoecologie.2462

ISSN : 2267-2419

Éditeur

Laboratoire Eco-anthropologie et Ethnobiologie

Référence électronique

Christian Seignobos, «L'arbuste Calotropis procera, un épisode de son histoire dans le bassin du lac Tchad », Revue d'ethnoécologie [En ligne], 9 | 2016, mis en ligne le 01 juillet 2016, consulté le 19 avril 2019. URL : http://journals.openedition.org/ethnoecologie/2462 ; DOI : 10.4000/ethnoecologie.2462

Ce document a été généré automatiquement le 19 avril 2019

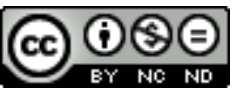

Revue d'ethnoécologie est mis à disposition selon les termes de la licence Creative Commons Attribution - Pas d'Utilisation Commerciale - Pas de Modification 4.0 International. 


\title{
L'arbuste Calotropis procera, un épisode de son histoire dans le bassin du lac Tchad
}

Calotropis procera, an episod of its story in the Lake Chad Basin

\author{
Christian Seignobos
}

1 Calotropis procera, le «roustonnier » du français argotique, «roustons » désignant les testicules, est également appelé "pommier de Sodome", la forme des fruits de cet arbuste évoquant un scrotum. Cette désignation est entrée dans le vocabulaire colonial comme le «saucissonnier » pour Kigelia africana, le « savonnier » pour Balanites aegyptiaca, le « baobab du chacal » ou le « pied d'éléphant » pour Adenium obesum... 


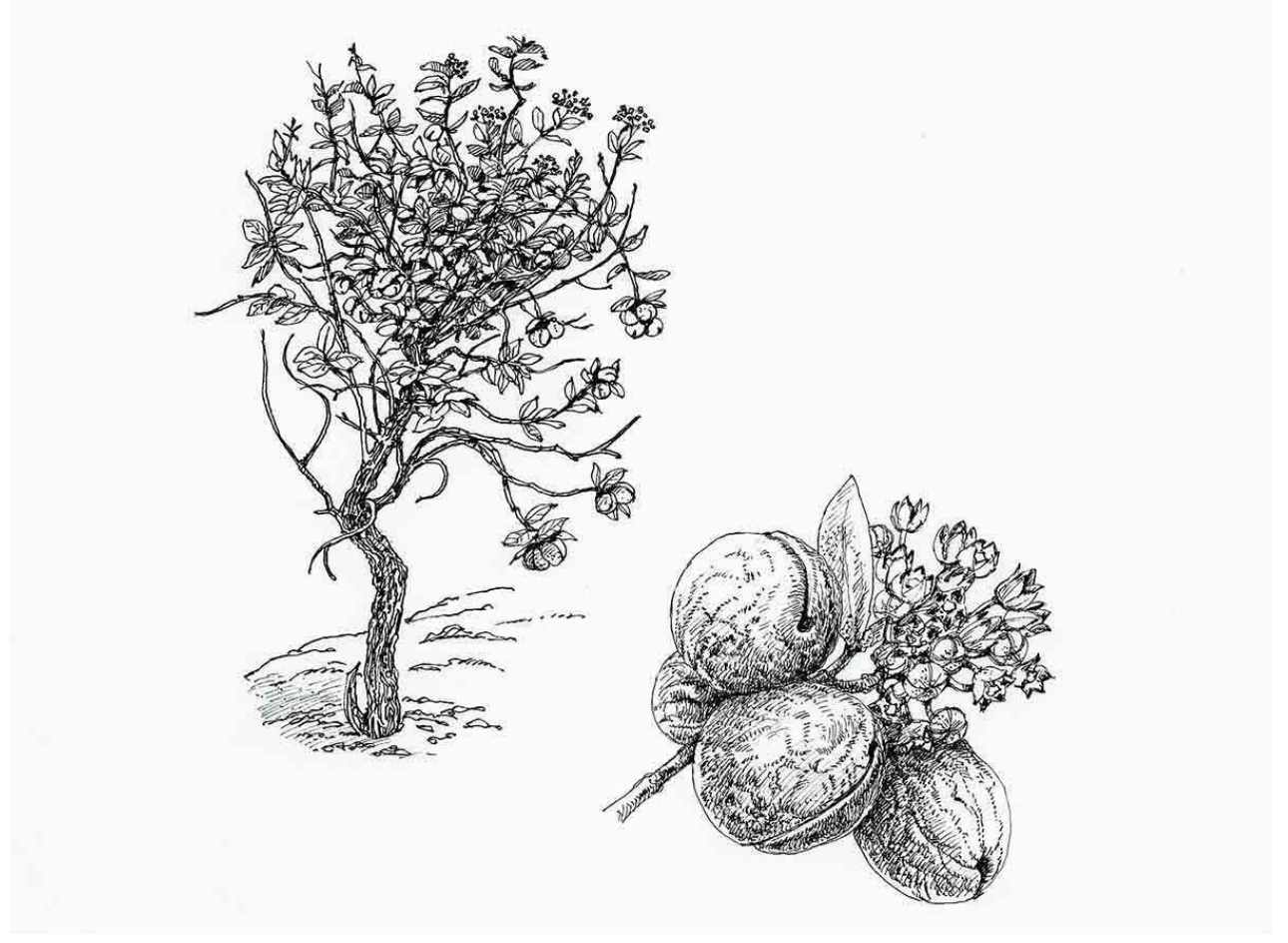

Dessin C. Seignobos

2 Cet arbuste a toujours intrigué le voyageur. A. Gide (2001: 246), passionné de botanique, en offre la description littéraire la plus accomplie lors de son séjour à Bol au nord du lac Tchad en février 1921 :

« Du sable, presque uniquement agrémenté par cette étrange plante gris-vert [Calotropis] [...], le fruit: un beignet énorme, bivalve, tenant suspendu en son centre, au milieu d'une matière feutrée, filigranée, un paquet de graines [...]. Rien de plus ingénieux et de plus bizarre. Les graines sont d'abord si étroitement juxtaposées, à la manière des tuiles d'un toit, que l'on ne soupçonne rien de ce duvet qu'elles protègent ; on ne voit d'abord qu'une carapace, une coque [...]. Dès qu'on presse cette coque, elle crève ; les graines se disjoignent, laissant paraitre un trésor soyeux [...], un émerveillement argenté qui tout aussitôt bouffe, foisonne, s'émancipe et se prépare à se laisser emporter au premier souffle $»^{1}$.

Dans ses Méharées, Th. Monod (1989 : 102) parle à propos de Calotropis de :

«[...] véritable provocation végétale en des lieux où on ne s'attendrait jamais à de telles apparitions [...], plante admirable de vigueur, de netteté, de franchise ; port résolument dressé [...], feuilles entières, arrondies, épaisses, charnues, vernies et comme laquées d'un vert légèrement bleuté, fleurs en corymbes terminaux, de velours mauve [...], fruit globuleux, allongé, vert, puis jaunâtre, contenant rangées à la façon des écailles d'un poisson, les graines, argentées d'abord, puis brunâtres... ».

H. Gillet (1968: 544) dans sa Note écologique et ethnobotanique sur Calotropis procera, signale que dans l'Ennedi, au Tchad, « les forêts de Calotropis » ont ceci en commun d'être installées « sur l'emplacement d'anciens campements de nomades ». Cette relation avec la matière organique fait (qu'à cette latitude?) «l'arbuste apparaît ainsi comme un psammophyte, anthropophile, nitratophile». Par ailleurs H. Gillet le présente en 
« colonisateur des terres ruinées » où les individus les plus prospères se remarqueraient sur les sols les plus pauvres, ce qui situe Calotropis hors de toute association végétale.

5 Pour E. Bernus (1981: 30), les peuplements de Calotropis commencent à devenir importants dans les régions du Niger, là où les autres espèces ont été éliminées par l'homme.

6 Dans les récits des coloniaux prenant pour cadre le sahel bien peu se sont abstenus de l'évoquer. Lors de sa première rencontre à Niormo, au sud d'Abéché, P. Fabre² (1933 : 247) le signale:

«Le jour s'est levé sur des vallonnements broussailleux, où régnait par endroits la verdure vénéneuse de l'arbuste « âcher », dont les larges feuilles sont gonflées d'un latex qui dégoutte abondamment lorsqu'on les blesse ou les casse». "Sid el Djouad » [le maitre du cheval, palefrenier] affirme que le lait de l'«âcher» rend aveugle le cheval dont la tête heurte par mégarde le feuillage débordant de poisons".

La dangerosité de Calotropis revient sans cesse dans les récits des coloniaux.

\section{Les modes de diffusion de Calotropis}

8 Tout l'appareil séminal de Calotropis est conçu pour voyager. Il faut avoir été spectateur du départ floconneux de ses graines portées par leurs fines aigrettes irisées, poussées par l'harmattan, les voir rouler à vive allure, sauter sur les sols dénudés ou encore emportées par les courants aériens pour ne plus douter de son pouvoir de dissémination. E. Bernus (1979: 120) signale la perception qu'ont les Touaregs Illabakan des mécanismes de cette dissémination. Les années de vents violents sur des couverts arborés et herbeux éclaircis par la sécheresse et offrant ainsi moins d'obstacles sont particulièrement favorables. Toutefois ce sont bien les grandes sécheresses, comme celle de 1973 et ses répliques de 1983-1984, qui ont relancé vers le sud la colonisation de sols épuisés qui, jusque là ne portaient que des pieds isolés de Calotropis sur les bords des routes qui les traversaient.

9 Cette diffusion est également redevable à l'homme par ses voyages, les transports depuis les caravanes d'âniers et de bœufs porteurs jusqu'aux camions, mais aussi les mouvements des éleveurs transhumants.

10 Calotropis semble s'être longtemps cantonné au nord du lac Tchad à des latitudes hautes, où il pouvait parfois se présenter en peuplements monospécifiques désignés sur les cartes des administrateurs coloniaux comme «forêts d'euphorbes ». Ignorant le taxon latin et n'osant nommer l'arbuste par «son nom obscène » ils parlent donc « d'euphorbes » par allusion à son latex (Seignobos \& Tourneux 2002: 51). Abou Digu'en (1929: 259), aventurier et négociant en bestiaux et en peaux, emploie lors de son séjour au Kanem le terme d' " oschards » (du nom arabe, " âcher », de Calotropis) ou de «fausses euphorbes ». Dans son livre «mes tournées au Tchad» le gouverneur P.-O. Lapie (1945: 206, 207), nourri des rapports de ses subordonnés, déclare s'être perdu aux abords du lac dans « les dédales des euphorbes ». Néanmoins les administrateurs vont, au cours de leurs tournées, multiplier les observations sur ces « champs d'euphorbes ». Très tôt ils signalent que ce curieux arbuste n'est pas inféodé à un milieu référencé mais suit le gradient de l'appauvrissement des sols. Ils rapportent aussi les nombreuses utilisations d'« âcher » par les indigènes. 
11 La chronique de la descente de Calotropis vers le sud se révèle malaisée à établir. Son envahissement de la rive méridionale du lac Tchad, comme dans la région de Katikimé, pourrait être récent sans toutefois nier une présence sporadique bien antérieure. Les cités kotoko seraient restées longtemps tributaires des Kanembu et des Buduma pour ce matériau de base de leurs filets de pêche. Les Kanembu filaient les fibres de Calotropis pour confectionner des cordelettes résistantes, aptes à subir des durées prolongées dans l'eau et jugées plus solides que celles issues des niébés textiles venus du sud. Des informateurs de Goulfey soutiennent que si les Kotoko étaient ainsi dépendants des gens $\mathrm{du}$ nord du lac c'est que Calotropis (peshi) était chez eux absent ou peu abondant. Blangwa, poste avancé kotoko sur le Bas Chari, les rives du lac étant peu sûres, recevait là des cargaisons de rouleaux de cordelettes apportées par les Buduma jusqu'à l'arrivée, au milieu du XIX ${ }^{e}$ siècle, des cordelettes d'Hibiscus cannabinus dont on crédite les Peuls venus de l'ouest de sa diffusion. Pour ces mêmes informateurs, une brusque poussée visible de Calotropis au sud du lac serait intervenue après l'épisode de sécheresse concomitant « avec l'arrivée des blancs » en 1900. Calotropis aurait encore cherché à coloniser en végétation pionnière le fond du lac asséché en 1908 :

«[...] steppe désolée, au sol gris-ardoise, profondément fendillé sous l'effet du soleil, où déjà la végétation arbustive est représentée par quelques oschars ( Calotropis procera)»(Carbou 1912 : 106).

G. Nachtigal, en 1869 au Bornou entre Kouka et Marte décrit une végétation monotone de palmiers doums et de Calotropis. Au Baguirmi $(1880: 371,372)$ il se plaint : "Hors des villes, ce sont les termites, qui y sont le cauchemar éternel du voyageur [...]. Veut-on camper en plein air [...] on dispose, si possible, tout autour du campement [...] certains branchages que l'insecte en question ne peut sentir. L'ochar [...] réputés souverains en ce cas ». Il livre cet aparté à Manjafa sur le Chari, mais rend-il compte de la situation présente ou des précédentes étapes plus septentrionales ? Le Calotropis à Manjafa signerait une présence déjà visible sur le Chari à la fin du XIX ${ }^{e}$ siècle.

13 En 1893, à Yola, capitale de l'Adamawa et aboutissement sur la Bénoué des routes caravanières, principalement du Bornou, S. Passarge $(2010: 566)$ signale cette Apocynaceae (sic).

14 Au Cameroun, à Maroua, sous le lamido Abduramani Suudi (1901-1908), des informateurs signalent que l'on n'osait déterrer Calotropis à cause de sa dangerosité, ce qui militerait pour une faible présence de cette Asclepiadaceae à cette époque.

15 Calotropis ne semble pas avoir participé à des descentes méridionales d'essences sahéliennes à des époques ante, comme Hyphaene thebaica par exemple. Le palmier doum a laissé des peuplements redevables à des formes de paléo-climats. On le retrouve en inclusions végétales résiduelles maintenues dans « des poches de sécheresse » selon J. Guillard (1965), dans la gouttière du Mayo Kebbi à la latitude de Léré comme sur les piémonts des Mandara au niveau de Méri, sur les hauts mayo Ranéo et Motorsolok.

Calotropis se présenterait comme un des meilleurs marqueurs de sahelisation de la zone soudanienne. Aussi G. Magrin (2000: 64) a-t-il raison d'enregistrer la présence de Calotropis pour renseigner cette descente du sahel. Au Tchad, il observait ainsi en janvier 1999 :

« les premiers Calotropis procera à une quarantaine de kilomètres au sud de Bongor, mais ils ne devenaient omniprésents qu'à une trentaine de kilomètres au sud de Guelengdeng ». 
17 Les grands axes routiers qui polarisent le peuplement et accroissent l'appauvrissement des sols tout en concentrant la circulation de véhicules et de bétail multiplient les facteurs de descente de Calotropis.

$\mathrm{Au}$ Cameroun, sur l'ensemble de l'axe du nord Maltam-Maroua-Figuil Calotropis est présent sur les bas côtés de la route et de là il se manifeste comme une espèce invasive sur les champs. Très ubiquiste il pousse sur les sols sableux comme sur les vertisols, jachères appauvries dans un cas comme dans l'autre. Calotropis pourrait faire partie du cortège de plantes post-culturales manifestant des caractères xériques plus accusés que les formations végétales primitives: Guiera senegalensis, Combretum spp., Piliostigma reticulatum... à ceci près qu'il n'aime pas la concurrence de recrû même peu dense. Cette singularité lui fait coloniser de préférence les terres usées. Sur les sols vertiques il se révèle plus dangereux à cause de son puissant système racinaire par lequel il pompe l'eau au détriment des sorghos repiqués, plus que ne le font les formations à Piliostigma reticulatum, pourtant souvent omniprésentes. L'extirper sur une parcelle relèverait d'un impossible combat.

19 Sur des itinéraires plus méridionaux on enregistre sa récente progression. À Ngong, au sud de Garoua, Calotropis se signale dans un grand nombre de cornières de cours d'habitations. En 2004-2005 il apparaît dans les champs proches des villages et également sporadiquement confiné aux talus de la route jusqu'à Mayo-Bokki. Plus au sud il ne se rencontre qu'à l'intérieur des cours, invité par le propriétaire. Absent au sud de Gouna il n'est visible chez les Dii que dans le village de Karna-Manga (2010). Encore inconnu sur le plateau de l'Adamawa il accompagnait dès avant 2000 certains périmètres de migrants venus depuis l'extrême Nord dans la région de Touboro ${ }^{3}$.

Comme pour tout phénomène de diffusion on se trouve confronté à la perception des interlocuteurs hésitant entre le cas index, celui d'une diffusion pionnière, et une vulgarisation qui marque le paysage. Mais partout il s'agit du même mode de progression. Les hommes implantent des pieds pionniers dans leur voisinage, leurs jardins de plantes médicamenteuses bien avant que quelques péjorations climatiques n'entraînent une diffusion spontanée de Calotropis toujours prêt à s'engouffrer, selon le mot de J. Guillard (1965 : 48) dans « une ligne de moindre résistance écologique».

Revue d'ethnoécologie, 9 | 2016 


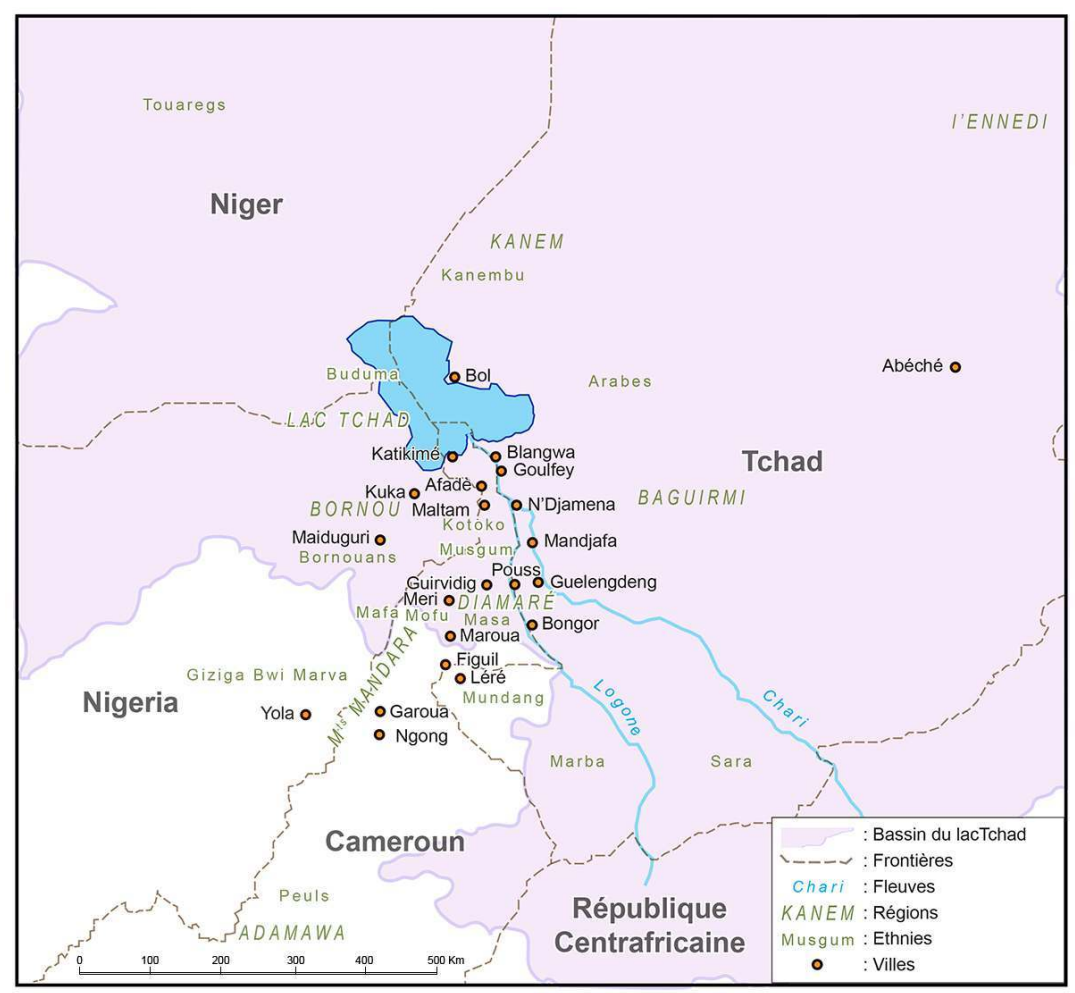

(c) L. Venot

\section{Le Calotropis au cœur des rituels}

21 L'homme peut agir plus directement encore dans cette diffusion car cette plante présente un trésor pour la pharmacopée et se trouve de surcroît accréditée de maints pouvoirs occultes.

R. Portères (1965 : 144), après avoir cité J. Kerharo et A. Bouquet (1950 : 194) au sujet des plantes médicales et toxiques, présente Calotropis comme «introduite par les féticheurs en raison de ses propriétés médicinales ». Il évoque aussi son rôle en protection des champs, des maisons « pour prévenir des tentations de sorcellerie ». Pour R. Portères :

« on est donc contraint de penser que Calotropis procera est une plante introduite en Afrique, en provenance de l'Asie, soit la voie arabo-islamique (peu probable), soit arabo-préislamique, et ayant gagné l'Atlantique par contact de magiciens d'Est en Ouest, ou bien à une époque encore plus ancienne. L'aire africaine actuelle ne serait qu'une expansion de l'aire asiatique originelle par les évocations et pratiques magiques ».

Le présent article ne peut qu'apporter crédit aux assertions de R. Portères car de semblables mécanismes se révèlent encore à l'œuvre dans la partie méridionale du circum tchadien. 


\section{Le Calotropis revendiqué dans les rituels identitaires chez les Giziga Bwi Marva de la région de Maroua} et d'autres moins avouables. Dans ces sociétés dominées par une culture de la sorcellerie la protection contre ses traits entraîne une recherche de leur détournement ou de leur renvoi à l'agresseur. Calotropis se veut une protection globale pour toutes les agressions venant de l'extérieur. Cela apparaît lors du rituel de fin d'année. Avant d'ouvrir la suivante les femmes balaient la concession avec des branches de Calotropis et elles iront jeter les balayures brûlées vers l'est, cérémonie appelée chez les Giziga comme chez les Mofu voisins : «crier le feu ». Kulfaya accompagne les différents rituels pour chasser les impuretés (madama) par des rogations autour de concessions, de villages, la population agitant des branches de Calotropis. Ce Calotropis domestique contrôle les femmes de la maisonnée dans leur va-et-vient à l'extérieur et assure la protection du grenier de réserve. Il est également assigné à la protection des enfants. Le chef de famille confectionne régulièrement une décoction de ses racines pour laver les enfants particulièrement vulnérables aux menées des sorciers ${ }^{4}$. On attache un petit faisceau de racines autour des reins de la femme enceinte qui auparavant a perdu des enfants. Le jour de l'accouchement, on le remettra au cou de l'enfant prolongeant ici son action protectrice. Enfin on enterrera des fleurs de Calotropis autour de la chambre de la jeune accouchée.

Le kulfaya est réputé comme un puissant kuli de protection contre le vol dans les maisons comme dans les champs et dans tous les conflits de voisinage. Les Giziga se moquent d'eux-mêmes en disant qu'ils ont passé leur vie à enfouir sur les limites de leurs parcelles des feuilles de kulfaya, avec de la cendre et des excréments humains. Mais cela correspond aussi à la réalité d'une insécurité foncière qui, ces deux dernières décennies, est allée croissant. Mais Calotropis sera surtout le medium absolu en cas d'épreuve judiciaire, l'ordalie (huduma vo : le jugement du corps). Ceux qui devront affronter les accusations d'adultère, de vol, de crime devant le jugement de kulfaya s'exposeront alors à la "maladie du kulfaya ", un gonflement de certaines parties du corps conduisant au trépas. La "maladie du ventre qui gonfle ", commune à de nombreuses ethnies, pourrait être imputée à plusieurs causes, celles relevant de sortilèges souvent de défunts parents mal intentionnés, celles nées du contact avec des objets ou des personnes impures mais 
surtout celles sanctionnant le résultat d'une ordalie. Invalidante socialement elle est particulièrement redoutée. Celui qui manifeste ces symptômes se voit coupé de sa communauté. On va même jusqu'à railler en public son ventre de batracien. À sa mort on l'enterre à l'écart et il se verra privé de funérailles. Ce mal pourrait être formellement identifié à des tumeurs, cirrhoses, parasites... les trocarts «à ponction d'ascite " étant inconnus jusqu'à la période coloniale, il entraînait en effet souvent la mort.

Le chef de famille prend soin de son Calotropis, sa «plante kuli ». Lorsqu'il prélève une branche pour la mettre sur ses récoltes dans les champs ou sur son silo, il explique son geste à l'arbuste. Après les battages il rapporte la branche à l'arbre (midiya kulfaya : la mère kulfaya) avec cette phrase : "Voilà ton enfant, le travail a été bien fait ».

Le Calotropis, toujours celui de la maison, permet de porter une agression occulte contre un tiers. Un Giziga brûle une branche et va enterrer les cendres près de la maison, généralement l'entrée, de celui dont il souhaite la perte. La famille visée voyant ses enfants tomber malades et après consultation des devins s'engage alors à régler, en chèvres et en linceuls (zana), le contentieux avec celui qui a déposé les charmes. Ce dernier ira alors récupérer, en cachette, les cendres de son kulfaya, avec quelques incantations codifiées.

L'utilisation de la branche ou des feuilles de Calotropis fonctionne soit pour mettre en branle le kuli, soit comme un exhausteur dans une démarche d'agression ou de protection engagée. Elle active une force dès lors irréversible après des sorts jetés avec d'autres cendres, un peu en guise d'intimidation (Guitard 2014 : 148).

31 Selon certains informateurs les feuilles disposées à l'entrée de l'habitation de celui qui se voit accusé de vol ou de sorcellerie sont devenues à ce point dangereuses que l'on ne peut ni les toucher ni même les enjamber... C'est un acte posé qui ne saurait être ignoré par la communauté villageoise. La famille visée ouvrira entre temps un autre passage dans la clôture de son habitation.

Nous avons pu suivre le fonctionnement du kuli kulfaya en $2008^{5}$. Dans le village giziga de Médemtéré au nord de Maroua, un homme avait disposé une branche de kulfaya pour protéger sa récolte de courges contre les voleurs. Ils survinrent, c'étaient des jeunes gens qui lui étaient apparentés. Lorsque sa femme meurt brutalement, les devins interprètent ce décès comme relevant bien du kuli kulfaya mais qui s'était retourné contre son commanditaire. Il ne manquerait pas de frapper indistinctement la famille comme s'il se trouvait déboussolé ne sachant qui choisir entre les différents agnats. Le diagnostic de « mauvaise mort » comparable à celle des suicidés empêche la famille de cette femme de l'enterrer. Le corps est confié à des forgerons fossoyeurs. Comme souvent dans la région les forgerons devenus les premiers "missionnaires", i.e. adeptes des missions, ici protestantes adventistes, déclinent la proposition. C'est alors qu'un enfant décède à son tour, déclenchant un grand désarroi dans toute la parentèle. La famille se réunit et, à travers force séances de devins et réunions de prières dans différentes chapelles, tenta de faire dévier la marche de ce kuli devenu incontrôlable.

L'intrusion des religions abrahamiques chez les Giziga a apporté, comme ailleurs, une donne perturbatrice. Le mouvement de profanation entrepris par les missions chrétiennes et par l'islam, jamais complètement achevé, n'en mine pas moins ces sociétés encore formellement dominées par une gérontocratie fortement misonéique. La « peur du sacré » entretenue autour du Calotropis a prolongé une sorte de croyance partagée, rendue toutefois moins univoque dans ses attendus. L'appartenance « missionnaire » n'ayant pas 
annihilé les rituels chez les paroissiens vieux adultes (« les pauvres en la foi »), elle les a rendu plus complexes.

De nombreux Giziga islamisés, christianisés et même demeurés païens, manifestent le désir de sortir du jugement par le kulfaya. La croyance n'est en rien émoussée, paradoxalement il s'agit plutôt du contraire. On craint que cette force immanente ne soit plus manipulable comme dans le passé. La communauté villageoise n'est plus unanime et une partie d'entre elle réside en ville. Comment dès lors pratiquer des ordalies, en tirer enseignement pour démasquer et punir?

Toutefois kulfaya, trop ancrée dans les institutions juridiques giziga, refait spontanément surface devant toute affaire à trancher. Pour mes interlocuteurs, il sera difficile de se passer du recours à kulfaya tant que le fond de sorcellerie ambiant se maintiendra.

«Missionnaires" et adeptes des kuley s'accordent néanmoins sur un point: pour déclencher une pareille force occulte il faut y être préparé, sous-entendu " mystiquement $»^{6}$. Avec kulfaya il s'agit d'une puissance ambivalente, vorace, quelle que soit la nature du conflit. Il faut savoir guider sa marche jusqu'aux fins désirées, l'empêcher de se muer en puissance perturbatrice néfaste et, enfin, éteindre son action mortifère. Les familles "dépassées " par de telles dérives font alors appel aux chefs, qui mobilisent les grands ritualistes, comme ce fut le cas dans l'affaire de Médemtéré.

Les chefferies giziga Bwi Marva (de second degré) comme celle du canton de Mambang ${ }^{7}$ disposent de deux types de référents juridiques. Les Giziga islamisés s'adressent à un alkali et jurent sur le Coran. Pour les «Julata » - ceux qui ne prient pas - le masahay, maitre de la terre, continue d'officier ${ }^{8}$. En cas d'accusation de vol et de meurtre on jure de son innocence avec kulfaya et on "mange le kuli» en mastiquant en public une de ses feuilles.

L'ordalie sublimale se déroule ainsi : l'accusé doit déterrer un Calotropis en présence d'une délégation conduite par le masahay. Il devra extirper toutes les racines sans jamais rompre la racine principale. Il effritera une à une les mottes de terre pour en libérer racines secondaires et radicelles. Si une racine se brise elle est mise dans l'eau et il boira ce macéré. Après cet exercice, s'il ne meurt pas au cours de l'année probatoire il est reconnu innocent. Une cérémonie de clôture confirmant l'arrêt du processus ordalique : buzluma kuli a kulfaya (ôter le kuli kulfaya) aura lieu chez le chef.

\section{Les pouvoirs de Calotropis, une croyance largement partagée}

Nous avons pu, fortuitement, en être témoin chez plusieurs groupes voisins des Giziga. J'ai assisté à un rituel proche de celui relevé chez les Giziga, intéressant Calotropis (tofome en mundang) en septembre 1997, au quartier de Sil Tigere dans le village de Gadas alors que j'exécutais le levé du terroir. Les Mundang se protègent des voleurs de récoltes sur pieds en pinçant deux feuilles de Calotropis dans une tige de mil fichée en bordure de la parcelle. Un habitant du village fut accusé de vol. Celui-ci furieux alla jeter deux feuilles de Calotropis dans la cour de son accusateur en disant haut et fort : «On va bien voir sur qui le mal va tomber!». Par ce geste l'accusé n'a fait que devancer la procédure ordinaire, devant le chef de terre (masay) où il aurait lancé sur son accusateur une branche de Calotropis et un fragment d'Euphorbia unispina (ndiye) en déclarant «Que le malheur me revienne si je suis l'auteur de cet acte ». Dans le cas d'une accusation à tort le Calotropis, convoqué pour désigner le coupable, peut se retourner contre l'accusateur. La 
famille du plaignant, effrayée par la tournure de l'affaire, promit de retirer sa plainte déposée auprès du chef. Les deux familles, l'accusée et l'accusatrice, appartenant toutes les deux à la mouvance missionnaire furent alors contraintes à se livrer à ce qui fut appelé un surga (travail collectif) pour déterrer le Calotropis auquel on avait soustrait les deux feuilles et cela sous le contrôle des représentants du chef et d'une délégation de villageois. Ce rituel commun a réuni une douzaine de personnes pendant plus de deux jours tant les racines de Calotropis sont profondes. Ce labeur serait le gage de leur réconciliation, toutefois l'idée d'annihiler toute poursuite de l'action engagée par le biais de Calotropis n'en demeurait pas moins présente. Le trou devait rester béant pour, me diton, servir d'exemple. La procédure serait donc manifestement didactique... Peut-on y voir le glissement d'une ordalie vers un rituel de réconciliation?

Figure 3 : Rite de conciliation. Mundang de Gadas

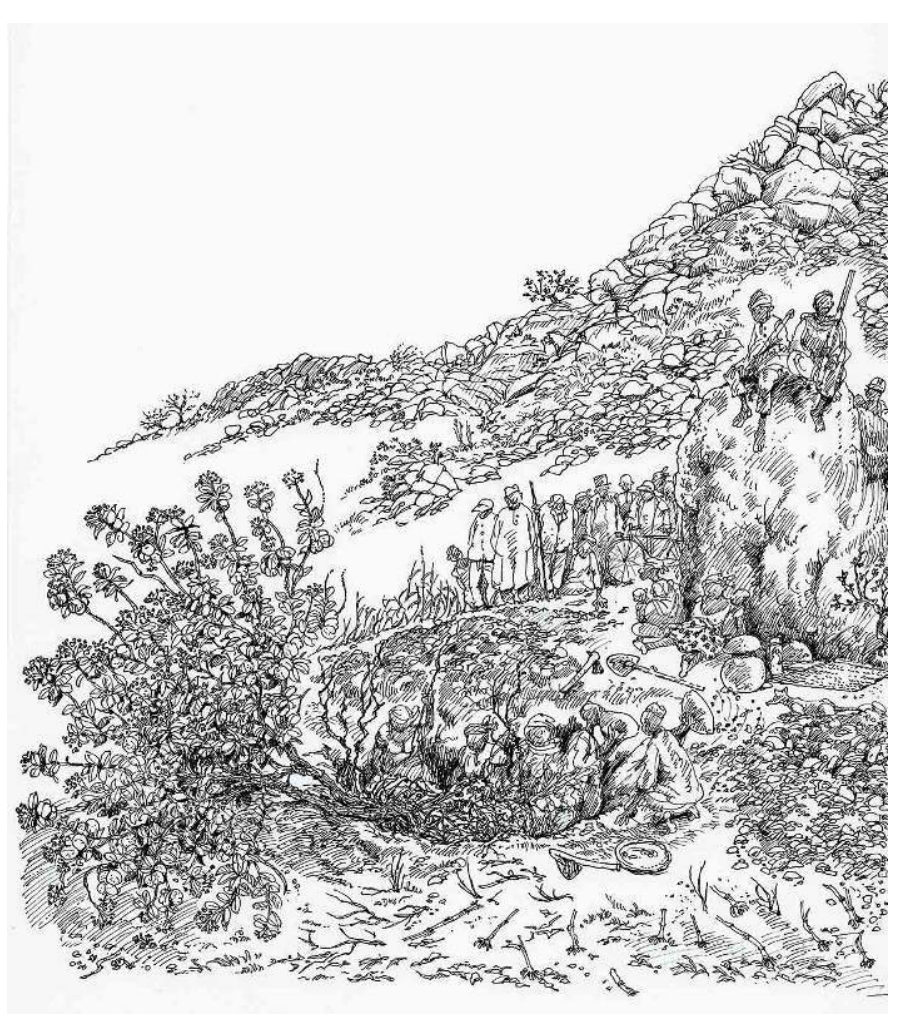

Dessin C. Seignobos

Ce rituel, dans son principe comme dans son déroulement, existe chez les Musgum Kalang (à Girvidig) et ceux de Pouss. On « fait croquer la feuille de Calotropis » (a luma muhur) à celui qui doit jurer de son innocence devant une accusation de vol, de meurtre ou de sorcellerie. Coupable il mourrait, et à sa suite les uns après les autres tous les membres de sa famille. La dangerosité du serment allait ici jusqu'à atteindre les témoins. À Pouss on dénonce également Calotropis comme un "médicament » doté d'une force telle qu'elle se révèle difficile à contrôler, pouvant devenir autonome et pernicieuse. Seul le recours à un rite puissant permet de se dégager de son emprise. On extirpe encore la racine pivot et aussi les extrémités racinaires du Calotropis dont on avait prélevé les feuilles pour le jugement. Les extrémités de toutes les racines, métaphore de la parentèle éloignée, seront données à consommer à chacun et leur amertume salvatrice fera fuir les maléfices. Puis l'ensemble des deux familles brûleront tout le Calotropis. De nombreux Musgum de Pouss 
ont été confrontés, dans leur jeunesse, à ce rituel, dont ils ont gardé le souvenir d'une atmosphère d'effroi. Dans les années 2000 l'islam, y compris dans ses formes intégristes, et le protestantisme ont pénétré ces communautés, aussi ces manifestations se seraientelles raréfiées.

41 À Maroua, ces pratiques des Giziga, perçus comme les autochtones de la ville, semblent avoir contaminé l'ensemble des communautés, celles demeurées païennes comme celles chrétiennes ou islamisées. Les Giziga étant réputés voleurs invétérés, leurs voisins, Peuls, Bornouans, Mofu disposaient alors des branches de Calotropis sur le moindre tas de mil, de coton ou de patates douces. Ils laissent aussi croitre à l'angle de leurs habitations un Calotropis à l'encontre des voleurs et toutes les sorcelleries en maraude.

Alors que j'enquêtais dans les saare à bil-bil (i.e. cabarets à bière de mil) en février 2002 à Maroua, j'assistais à une scène mettant en cause Calotropis. Dans un cabaret fameux, «le cabaret Zazou ", parmi les mieux achalandés du grand quartier à bière de Domayo PontVert, un grand Calotropis protecteur trônait à côté de «l'usine », partie de la concession où se brasse la bière.

Figure 4 : Cabaret à bière, Maroua

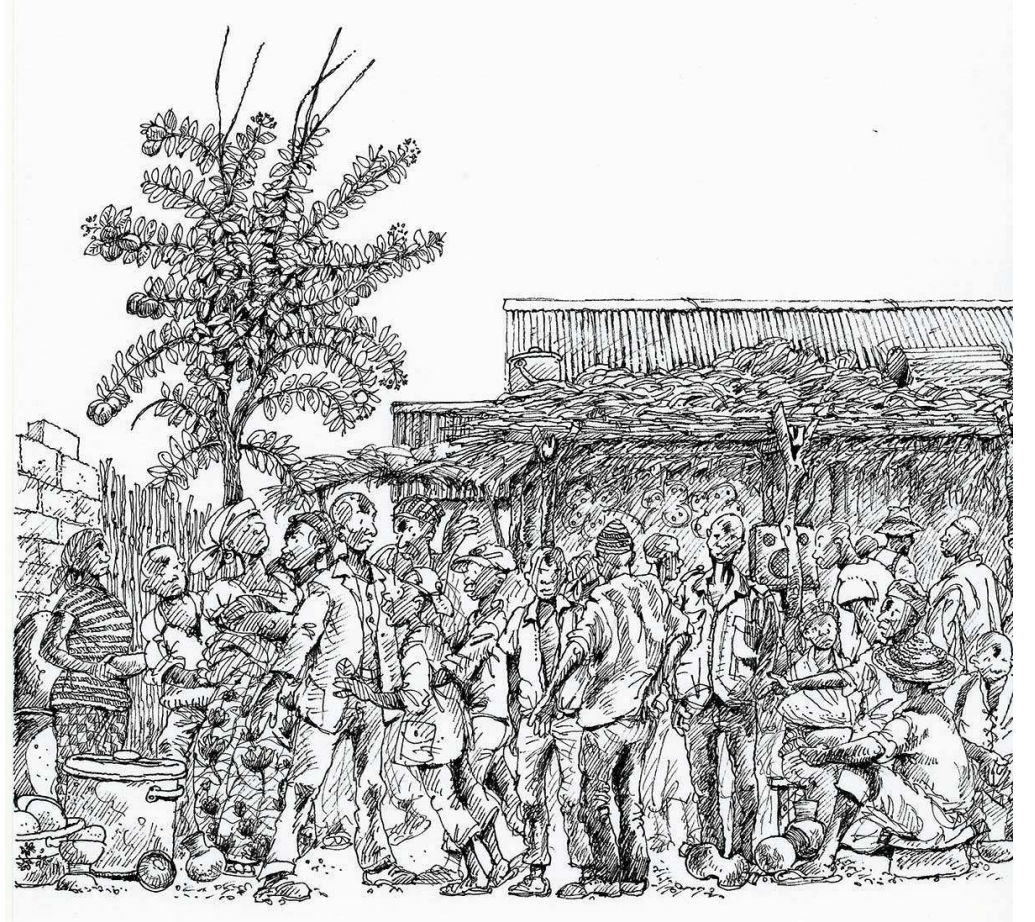

Dessin C. Seignobos

Un dénommé Bouba, un jeune "clando ", taxi-moto, de son état, après avoir bu sa bière entre deux courses et payé avec une pièce de $500 \mathrm{Fcfa}$ attend sa monnaie. La tenancière, occupée auprès d'autres clients, l'oublie. Le jeune taxi-moto réclame sa monnaie, mais Madame Zazou lui dit n'avoir rien reçu de lui, ni pièce de 100, ni de 500. Furieux le jeune homme se précipite sur le kulfaya et tend alors une pièce pliée dans une feuille de Calotropis, qu'il veut obliger la cabaretière à prendre. Des clients s'interposent pour que Madame Zazou n'en fasse rien. Devant cette forme sauvage d'ordalie la situation est jugée grave. On envoie chercher le baaba lawaale qui règle les conflits dans les cabarets de 
chaque quartier à bière. Il est absent. Tout le saare à bil-bil, soit ce jour-là une quarantaine de personnes, est en émoi. Deux clients décident de payer la tenancière et demande expressément à Bouba d'aller enterrer la feuille de kulfaya pour clore l'affaire. Cet incident démontrait que tous les participants étaient avertis du rôle de l'arbuste dans les jugements et de sa dangerosité. Les protagonistes ne sont pas giziga. Bouba est un Mofu Duvangar, et Madame Zazou, si sa mère est giziga, son père est tupuri. Quant aux clients qui ont bien voulu payer pour Bouba, l'un est sara, l'autre mafa.

Calotropis est une manifestation forte de ces hybridations d'héritages cognitifs dont rend compte toute cohabitation urbaine.

On peut être tenté de faire l'historisation des rituels concernant Calotropis procera. Ils viendraient du nord, les Kotoko d'Afadé et ceux de Makari les auraient pratiqués et avant eux les Bornouans. Via les Mandara ils gagnent les pays musgum et giziga. Alors que l'islam, le rouleau compresseur des paléocroyances, efface ces pratiques ordaliques'. Le Calotropis « porteur de sacré » descend toujours plus vers le sud. Les Giziga Bwi Marva en assurent la promotion. Ces pratiques touchent les Mundang et les Masa s'arrêtant sur cet ultime front de descente du Calotropis lui-même. Les lignes d'isocroyances concernant Calotropis ont ainsi évolué dans le temps en se déplaçant vers le sud.

Selon nos informateurs l'apparition de ces pratiques ne pouvait se réaliser qu'à des époques où Calotropis était peu abondant. Ainsi le rite de l'extraction totale de l'appareil racinaire, en contradiction formelle d'interdire l'abattage sans raison de cet arbuste, renforçait-il ce geste. Calotropis va peu à peu remplacer dans le sud une plante à latex, Euphorbia unispina, qui sert de protection occulte et pour les ordalies. Les deux plantes peuvent encore " coopérer » dans certains groupes mundang et masa alors que, plus au sud, chez les Marba et les Musey, on s'en tient à la seule Euphorbia unispina. Dans le pays masa, Caloropis (furuda) a pris le nom de l'Euphorbia unispina (furu). Cette dernière plante a dû être requalifiée en furu ma jufna (Calotropis / qui / mâle) (Melis 2006: 142). La mention «mâle » apposée au nom de la plante est une marque d'antériorité dans toutes les langues de la région pour distinguer deux espèces présentant quelques ressemblances et dont on requiert les mêmes services.

\section{Les mille et un usages de Calotropis}

Lorsqu'on questionne sur le rôle de Calotropis, les réponses sont très spontanées, avec les expressions évocatrices du français local, que nous avons choisi de garder. La première est immanquablement : « il ne faut pas prendre cet arbuste à la légère, banbambe ${ }^{10}$ peut nous dépasser " et les mêmes informateurs de préciser : "Il est interdit de s'amuser hasardement avec les feuilles de cette plante car les hommes doivent régler leurs problèmes avec elles». On ne peut pas faire entrer du bois de Calotropis dans une concession et le brûler dans un foyer, "c'est une plante pour les sacrifices » et cela provoquerait la mort du chef de famille. On relève encore : "c'est un arbre qui pousse naturellement sacré », ce qui sous-entend que les autres ne le deviennent qu'avec le temps. En effet on abat difficilement de gros arbres âgés, même chez les musulmans, car ils sont suspectés d'héberger quelques génies. Banbambe n'est-il pas considéré comme le djinn des autres arbres, et jadis on ne pouvait le déterrer sans précautions rituelles.

On relève d'autres assertions assez voisines «banbambe est né médicament » (lekki) ${ }^{11}$, autrement dit, en dépit de son abondance actuelle il reste un lekki efficace. Cela infirme 
le cheminement couramment observé d'une plante, cultivars compris, introduite souvent sous un usage médicinal, qui, après sa vulgarisation, perd ses qualités premières. On a pu l'observer avec l'introduction d'Ipomoea fistulosa dans les années 1930. Appartenant à la catégorie des gabde makka (végétal/La Mecque) elle a été "acclimatée " dans les concessions de la région de Maiduguri (Bornou) comme plante médicinale et faisant fuir les serpents. Rapidement sortie des habitations elle s'est naturalisée un peu partout, ce qu'explique son appellation tigga-miccito (on bouture et on le regrette). Entre temps cette Convolvulaceae avait perdu les vertus premières qu'on lui prêtait.

\section{Utilisations formelles et pharmacopée}

Dans l'ouvrage de Dalziel The Useful Plants of West Tropical Africa (1948 : 384-386), Calotropis procera constitue une, sinon la plus grosse entrée de cette somme.

Nous signalerons certaines utilisations concernant banbambe. Lorsque ses feuilles d'un vert malpropre, empoussiéré à la fin de la saison sèche ont été lavées par les premières pluies et que de nouvelles feuilles apparaissent aux extrémités elles peuvent être broutées par les chèvres qui, de leurs pattes avant, font fléchir la tige principale. Autour du lac Tchad, là où les arbres ont disparu, le Calotropis constitue la principale ressource ligneuse, redoublant son aspect invasif. Au Kanem il est utilisé en vif pour des clôtures. Le bois sert dans la fabrication des toitures d'argamasse (toits plats en terre). On aligne sur les poutres les bois de Calotropis en chevrons serrés. Épargnés par les termites ils soutiennent le revêtement de la toiture. Ses branches souples sont également requises pour les tores des toitures coniques sur lesquels on liera les perches.

51 Après avoir recouvert de vannerie les silos souterrains on y étendra une couche de feuilles de Calotropis avant d'y verser le sable. Grâce au latex qui les lie entre elles, elles assureront une fermeture hermétique contre les infiltrations. À Goudoum-Goudoum, dans le Diamaré (mai 2001), lors de la fermeture d'un de ces silos souterrains et alors qu'un plastique devait permettre l'imperméabilité, le propriétaire a tenu à rajouter quelques feuilles de Calotropis... contre les voleurs.

Le charbon de bois de Calotropis mélangé à du soufre et du salpêtre compose la poudre pour les fusils de traite. On l'utilise également pour l'encre contenue dans les encriers des élèves coraniques. Cette encre apporterait un adjuvant bénéfique aux sourates écrites sur les planchettes coraniques que l'on lavera et dont on boira l'eau.

Le carquois en Calotropis, bois dur et léger est affectionné par les chasseurs professionnels (gaw). Le poison des flèches s'altérerait moins à l'intérieur. On utilise également les feuilles fraîches pour donner la forme de l'arc à double courbure...

Les utilisations les plus courantes de Calotropis restent celle d'emballage de produits et de nourritures. On place les grains de sorgho mis à germer pour la confection de la bière sous des feuilles de banbambe qui entretiennent l'humidité recherchée. On mouille la farine des fruits de jujubier que l'on enveloppe de feuilles de Calotropis avant de la mettre dans une poterie sur le foyer où elle prendra forme. Cette galette solide (yaa'baande) est commercialisée sur les marchés. Ces feuilles sont également requises dans la fabrication de petits pains de sel.

Les Calotropis issus de défrichements des vertisols sont mis à sécher puis brûlés. La cendre donnera, après lixiviation, un sel de potasse apprécié. Dans le «classement » de ces sels aqueux de la région de Maroua il arriverait en troisième position pour le goût après ceux 
de Balanites et des tiges de petit mil. Toutefois la cendre de Calotropis présente un autre intérêt, celui de "coupage» avec d'autres cendres afin de les bonifier. L'une des combinaisons les plus appréciées serait l'association d'un tiers de cendre de Calotropis pour deux tiers de cendre de cannes de sorgos rouges (Langlois et al. $2013: 287$ ) (...).

De nombreux auteurs s'accordent à présenter cette Asclepiadaceae comme une véritable base de produits à verser dans différents compartiments de la pharmacopée. Th. Monod signale, dans Méharées, la migration de substances toxiques du Calotropis : la calotropine et la calactine à travers la chenille d'un certain papillon (Danaus plexippus) qui, ayant ingéré des substances toxiques, engendre des imagos toxiques pour les oiseaux qui en les ingérant s'intoxiquent à leur tour. Les Mofu Gudur désignent le "criquet puant", débauche de couleurs, Zonocerus variegatus, comme « le criquet/Calotropis» toxique par sa proximité avec la plante hôte (Barreteau 1999: 148). Un autre criquet jaune pâle, légèrement moucheté, Poekilocerus bufonis hieroglyphicus, inféodé lui aussi aux Asclepiadaceae, appelé " criquet/poison ", entretient la même réputation. Au contact de Calotropis ces deux criquets ne seront pas consommés et serviront d'additifs à certains poisons sagittaires.

"L'ingestion des fleurs [de Calotropis] est pratiquée pour chasser les vers intestinaux. Les fruits cuits avec du mil rouge et des racines cuites avec du mil chandelle sont réputés pour redonner des forces aux vieillards. La racine est utilisée comme vomitif contre la syphilis!».

A. Vaillant (1945: 44), ingénieur d'agriculture coloniale à Maroua semble encore en exagérer l'efficacité :

«[Calotropis procera] exsude un latex caustique (calotropine), dont le principe actif est plus puissant que le Strophantus et se conserve assez longtemps à l'état desséché. Sert comme poison de flèche ${ }^{12}$ et poison d'épreuve. L'écorce des racines est médicamenteuse et renferme des principes amers (mudarine ou asclépine) [...]. Le latex est utilisé pour le traitement de la gonorrhée et de la syphilis ».

La médication suit ici parfaitement la théorie des signatures : le fruit de Calotropis comme scroton...

Une majorité de soins passe, en effet, par le latex de Calotropis, souvent appelé, comme chez les Giziga, la larme de Calotropis (t'way a kulfaya). Pour les intéressés « c'est comme l'alcool à $90^{\circ}$ ». On soigne aussi et cautérise les plaies avec les feuilles jouant le rôle d'antiseptique. Elles se collent entre elles, et fournissent de meilleurs pansements que celles de Combretum glutinosum (buski) ou de Combretum molle (sankiita saare). Ces feuilles enraieraient même le tétanos! Pour les plaies de saison sèche, «celles qui ne guérissent pas " par opposition à celles de saison des pluies, plus conjoncturelles, on emploie alors les racines de Calotropis broyées dans quelques égrugeoirs. Pour la réduction des fractures on appose des cataplasmes de feuilles chauffées sur des applications préalables de beurre ou d'huile. Partout les feuilles de banbambe mises sur des braises puis sur la partie malade « calment la douleur ». Chez les Mofu les feuilles de Calotropis chauffées sont appliquées sur les brûlures, chez les Masa elles soignent les morsures de serpent, ailleurs plutôt les piqûres de scorpions. On utilise le latex contre la lèpre, mais toujours associé à l'écorce de Sterculia setigera (bo'bori), le grand référent dans tout le bassin du lac Tchad pour traiter cette maladie. On soigne la varicelle (ngaadiga) en frottant le corps avec le liquide issu de la lixiviation des cendres de Calotropis ${ }^{13}$. Ce même filtrat, convenablement dosé, est donné aux enfants atteints de rhumes et de 
quintes de toux. Enfin les macérations ou les décoctions de racines servent de multiples applications médicales, comme vermifuge, diurétique...

61 Au Cameroun, la pharmacopée vétérinaire la plus développée concerne les équidés. Elle possède dans le Diamaré des spécialistes, des Peuls Suudu Dembo (affranchis et intronisateurs des Ardo'en ngara de Pété) comme ceux résidant à Balaza-Domayo et qui, depuis des lustres, élèvent et soignent les chevaux. Ils traitent le murgude, mal de dos des chevaux, piquant les reins à l'aide d'un petit poinçon tout en y appliquant du beurre fondu. Des feuilles de Calotropis chauffées recouvrent ensuite cette partie de l'animal à la façon d'un cataplasme, plusieurs fois renouvelé. Ils soignent également tuusaare (ga'e puccu : la syphilis du cheval), en fait la lymphangite épizootique des équins. Dès leur apparition les pustules sont percées avec des épines de Balanites que l'on aura auparavant laissées durant trois jours plantées dans le tronc d'un banbambe pour recueillir le pouvoir de sa sève. Calotropis n'est requis qu'en soins externes.

Les Musey soignent leurs poneys avec de la poudre de feuilles de Calotropis sur les plaies dorsales. Ils utilisent la sève pour faire cicatriser les plaies des bubons provoqués par la morve ou à la dourine en renouvelant les applications tous les trois jours. Le Calotropis étant peu présent dans le paysage, ces posologies ont été empruntées aux Peuls.

Une pommade composée de beurre et d'un macéré de racines de Calotropis est requise contre la gale des petits ruminants. E. Bernus $(1981: 193)$ le signale également chez les Touaregs du Niger. Dans les bergeries infestées de puces, après balayage du sol, on brûle des feuilles de Combretum glutinosum avant d'étaler un tapis de feuilles de banbambe, dont « l'odeur » doit tuer les puces.

Pour favoriser la fécondité du bétail chez les Giziga et certains Musgum on attache les brebis chacune à une branche de Calotropis plantée au-dessus d'un lot de quatre-vingt-dixneuf fruits de Ziziphus mucronata, l'épineux aux épines réputées les plus accrocheuses, mis en terre. Mais nous entrons là dans l'infini registre des charmes...

\section{Calotropis : le côté sombre de la force} chroniqueurs, comme un grand illusionniste qui joue de son étrangeté et partant des pouvoirs qu'on lui prête.

\section{Al-Bakri, pour ne citer que lui, signale en 1068 le Calotropis :}

«parmi les merveilles du pays des Sudan [...]. Il pousse dans le sable et porte un gros fruit, garni d'une sorte de laine blanche, utilisée pour la confection de vêtements et de tissus qui résistent au feu le plus ardent » (Cuoq 1975 : 104).

Les informations auraient été recueillies au Ghâna, au sud de l'actuelle Mauritanie. A-t-on effectivement filé et tissé la « laine » i.e. les aigrettes attachées aux graines de Calotropis ? À moins qu'il ne s'agisse du rembourrage de quelques armures matelassées? Cette propriété ignifuge est d'autant plus surprenante que la «soie/laine» de Calotropis se voit partout reconnue comme un excellent amadou. Les fibres issues du liber, en revanche, partout tressées en cordelettes, auraient pu être tissées. R. Mauny (1961: 235) dit avoir fait l'expérience du caractère non ignifugé de la plante. Toutefois la prétendue propriété d'amiante de la « laine » ne relèverait-elle plutôt du registre des protections occultes ?

Peu d'essences ont autant fait l'objet de manipulations occultes que Calotropis de la fleur mauve pâle en capitule à la racine rougeâtre via sa feuille charnue, son écorce subéreuse, 
son latex éclatant et jusqu'aux Loranthus qui le parasitent. Même Piliostigma reticulatum, barkehi (l'arbre de la barka) des Peuls n'a jamais répondu à autant de sollicitations.

Les recettes relevées multiplient à l'envi le choix des jours, des heures de cueillette, des orientations, des chiffres concernant les graines, les feuilles à prélever et des ingrédients d'accompagnement. Se mêlent héritages musulmans et pratiques païennes pour des usages occultes passant par tous les modes d'administration possibles, macération, potion, décoction, lixiviation, fumigation...

Calotropis n'est pas pour autant un medium neutre comme le sont les Cissus quadrangularis ou les géophytes de type Crinum, patrimoine familial, qui se bouturent, s'héritent, s'échangent et s'achètent. Ils se montrent capables d'endosser n'importe quel charme pour n'importe quelle posologie. Calotropis représente, nous l'avons vu, une force difficile à domestiquer, principalement dans son rôle judiciaire. Il répond néanmoins aussi à des sollicitations domestiques. Les paysans giziga Bwi Marva, par exemple, enduisent, avant chaque campagne agricole, le manche de leur houe avec du latex de Calotropis. Il se prête surtout à la rédaction de "traités » concernant la fabrication de phylactères, philtres, charmes, pour des "blindages", des enrichissements, l'obtention de faveurs... avec des formules aussi alambiquées que celles que l'on peut recueillir auprès de chasseurs professionnels (Seignobos 2011).

71 Nous ne mentionnerons que quelques exemples pris parmi des communautés islamisées. Tout ou partie de Calotropis, y compris le charbon, est retenu pour des formules de «blindage ». Les épiphytes, assez rares il est vrai, sont particulièrement recherchés pour des phylactères contre les armes blanches. Les graines préparées avec celles de sorghos rouges seront ingérées régulièrement pour entretenir un "blindage » initial à base de Calotropis. Satisfaire un désir de lucre et de réussite peut aboutir aux démarches les plus saugrenues. Dans la Bénoué, un « rituel d'enrichissement » recommande de se rendre nu, la nuit, pour accrocher des lanières de viande sur un Calotropis. Une fois sa demande formulée, il faut fuir sans se retourner. Par la suite il n'est pas rare d'être sujet à des hallucinations... Certains reviennent, toujours nuitamment, reprendre cette viande pour la consommer ${ }^{14}$. La quintessence de la puissance du Calotropis tient à ses racines. Un quidam peut déterrer une racine traçante d'un banbambe, il la coupe et la réintroduit intégralement dans l'autres sens, l'extrémité près de la souche et la recouvre de terre. Il revient une semaine plus tard, toujours la nuit, tout en dominant sa peur car quelques prodiges peuvent se manifester. Il emporte la racine chez lui, ce puissant «remède » répondra à bien des attentes.

On cueille les fruits de Calotropis choisis à partir de sept pieds différents et que l'on fait éclater. Ceux qui ne font pas de bruit sont conservés. Les graines sont ingérées selon un certain rythme. Les enveloppes des fruits, séchées puis pilées avec des racines d'Abrus precatorius (belemhi) et plongées dans du lait caillé seront bues. Le but: détourner de soi le mauvais sort, l'injustice et s'attirer les bonnes grâces de puissants. Une démarche semblable intéresse la lutte contre les sorciers. Sur sept pieds de Calotropis on prélève des fragments de racines situées dans l'ombre portée de l'impétrant, on les écrase avec celles d'indigotiers, de Cassia occidentalis (kaccu-kaccunga) et de elelihi, toutes plantes réputées efficaces contre la sorcellerie. Puis on prépare une décoction... 


\section{Conclusion}

73 Calotropis semble avoir eu sur les hommes l'étrange pouvoir de stimuler leur imagination. Il exalte une fantasmagorie liée à ses attributs, renforcée par les mouvements d'ombres et de lumières ou encore $\mathrm{du}$ jeu du vent nocturne. Il émanerait de lui des signaux acoustiques, chuchotements, paroles étouffées...tels que prêtés aux humains. Même P. Fabre (1935: 181), autour d'Abéché, parle des «euphorbes arborescentes» qui la nuit « semblent rôder autour des promeneurs, en des clartés de souterraines aurores ». On fait encore peur aux enfants en rappelant que la nuit les Calotropis se transforment en fantômes et en ogres pour hâter leur retour à la maison. Leurs silhouettes effraient non seulement les enfants et les ivrognes qui se sont attardés dans quelque marché à bière mais elles poussent à des visions hallucinatoires. À Balaza-Alkali on se gausse encore d'un chasseur (gaw) de passage la nuit il y a quelques décennies, qui, à la sortie du village, croyant être assailli par des coupeurs de route aurait vidé le contenu de son carquois sur un groupe de Calotropis.

Ses attributs, son fruit en forme de scrotum et son latex ont toujours alimenté une grande fécondité métaphorique. L'extraordinaire, pour Calotropis, est d'avoir conservé intacts à la fois l'épouvante et l'espoir qu'il inspire sur un temps aussi long. Ce sont bien sûr aussi les services rendus dans le domaine des soins et dans celui infini des activités occultes qui ont, durant des générations, animé les chroniques judiciaires. Ce constat éclaire la diffusion de ce végétal singulier dans sa descente méridionale avant de voir en lui les stigmates d'une désertification en marche.

\section{BIBLIOGRAPHIE}

Abou Digu'en 1929 - Mon voyage au Soudan Tchadien. Paris, 291 p.

Barreteau D. 1999 - Les Mofu-Gudur et leurs criquets. In Baroin C. \& Boutrais J. (Ed.) L'homme et

l'animal dans le bassin du lac Tchad. Ird : 133-169. (Colloques et Séminaires).

Bernus E. 1979 - L'arbre et le nomade. Jatba XXVI (2) : 103-128.

Bernus E. 1981 - Touaregs nigériens, unité culturelle et diversité régionale d'un peuple pasteur. Orstom, 507 p. (Mémoire ; 94).

Carbou H. 1912 - La région du Tchad et du Ouadaï. T1 Paris, E. Leroux, 380 p.

Cuoq J.-M. 1975 - Recueil des sources arabes concernant l'Afrique occidentale du VIII au XVI (Bilad al Sudan). Paris, CNRS, 490 p.

Dalziel J.M. - The useful plants of West tropical Africa. London, Westminster, 612 p.

Fabre P. 1933 - La randonnée. Marseille, Éditions des Cahiers du Sud, 262 p.

Fabre P. 1935 - Les heures d'Abéché. Marseille, Éditions des Cahiers du Sud, 280 p. 
Gide A. 2001 - Voyage au Congo, suivi de Le retour du Tchad, (Carnets de route). Paris, Gallimard, 554 p. (Folio).

Gillet H. 1968 - Note écologique et ethno-botanique sur Calotropis procera. Jatba XV (12) : 543-545.

Guillard J. 1965 - Golonpoui, analyse des conditions de modernisation d'un village du nord-Cameroun. Paris/La haye, Mouton, $502 \mathrm{p}$.

Guitard E. 2014 - « Le grand chef doit être comme le grand tas d'ordures ", Gestion des déchets et relations de pouvoir dans les villes de Garoua et Maroua (Cameroun). Thèse Université Paris-Ouest La Défense en Anthropologie, $600 \mathrm{p}$.

Jullien de Pommerol P. 1999 - Dictionnaire arabe tchadien-français. Paris, Karthala, 1640 p.

Kerharo J. \& Bouquet A. 1950 - Plantes médicinales et toxiques de la Côte d'Ivoire-Haute-Volta. Paris, Vigot, $295 \mathrm{p}$.

Langlois O., Seignobos C. \& Anderson P. 2013 - Vers une histoire du « sel de potasse » dans le nord du Cameroun : observations préliminaires. In Anderson P.C., Cheval C. \& Durand A. (Ed.) Regards croisés sur les outils liés au travail des végétaux, XXXIII ${ }^{e}$ Rencontres internationales d'archéologie et d'histoire d'Antibes : 279-295.

Lapie P.O. 1945 - Mes tournées au Tchad. Alger, Office français d'éditions, 283 p.

Magrin G. 2000 - Le sud du Tchad en mutation, des champs de coton aux sirènes de l'or noir, T1. Thèse Université Paris 1 Panthéon Sorbonne, $604 \mathrm{p}$.

Malzy P. 1954 - Quelques plantes du Nord Cameroun et leurs utilisations. Jatba 1 (5-6) : 148-179.

Mauny R. 1961 - Tableau géographique de l'Ouest africain au Moyen-âge d'après les sources écrites, la tradition et l'archéologie. Dakar, Ifan, $587 \mathrm{p}$.

Melis A. 2006 - Dictionnaire Masa-Français, dialectes Gumay et Haara (Tchad). Editrice Democratica Sarda, $393 \mathrm{p}$.

Monod T. 1989 - Méharées, explorations au vrai Sahara. Arles, Actes Sud-Babel, 333 p.

Nachtigal G. 1880 - Voyage du Bornou au Baguirmi. In Le Tour du Monde. Paris, Librairie Hachette et $C^{\text {ie }}: 337-416$.

Passarge S. 2010 - Adamawa. Rapport de l'expédition du comité allemand pour le Cameroun au cours des années 1893-1894. Traduit de l'allemand et présenté par Mohammadou Eldridge. Paris, Karthala, $622 \mathrm{p}$.

Portères R. 1965 - Le caractère magique originel des haies vives et de leurs constituants (Europe et Afrique occidentale). Jatba XII (6-7-8).

Seignobos C. \& Tourneux H. 2002 - Le Nord-Cameroun à travers ses mots. Dictionnaire de termes anciens et modernes. Paris, Ird-Karthala, $334 \mathrm{p}$.

Seignobos C. 2011 - Les gaw du Cameroun, de chasseurs à tradipraticiens. Journal des Africanistes 81 (1) : 35-59.

Vaillant A. 1945 - La flore méridionale du lac Tchad. Bulletin de la Société d'Études camerounaises 9 : 13-14. 


\section{NOTES}

1. Les aigrettes attachées aux graines, évanescentes et argentées, pouvaient évoquer la légèreté et la brillance de la soie, d'où le nom de « arbre à soie du Sénégal » parfois donné à Calotropis.

2. Premier fonctionnaire civil à Abéché $P$. Fabre fonda une école après la guerre de 14-18. Il traversa le Tchad à pied, à cheval et en bœuf porteur. Il décrit son voyage dans « La randonnée ", ouvrage malheureusement peu connu.

3. À Bafia, bourg au contact forêt/savane, dans le quartier hausa, on note en 2010 dans les concessions un panel de flore sahélienne: henné, jujubier, Moringa oleifera, tamarinier et Calotropis.

4. Il s'agit de sorciers " anthropophages ", mujuuri.

5. Informateurs : Paul Djouldé Adama (Maroua) et Ali Dawa (Médemtéré).

6. «Mystique » est depuis deux décennies régulièrement employé dans les discours en français. Accolé à une personne le terme n'est pas forcément négatif, il renvoie à des pouvoirs ésotériques captés éventuellement après un cheminement plus ou moins long, parfois à travers plusieurs cultures.

7. Le chef de canton Wassili Hamidou (décembre 2008) fut un de nos principaux informateurs, quant aux enquêtes dans l'aire de peuplement giziga Bwi Marva, mentionnons également, entre 2000 et 2008, Njidda Kitikil, Douboula Amani et Akouli Bouba de Kossewa; Hamadou Bouba à Jebbé et Yaya Gournay à Houloum-Marva Maliki Wassili était notre interprète.

8. Les chrétiens appartiennent généralement à une autre sphère juridique. Issus de la scolarisation ils s'adressent aux tribunaux publics souvent après avoir tâté de la justice traditionnelle.

9. On peut en retrouver des bribes concernant le Calotropis auprès de divers groupes musulmans. Chez les Mandara et divers Foulbéisés, les gens suspectés de sorcellerie sont fouettés à l'aide de branches de Calotropis. Seuls ces fouets peuvent les contraindre à libérer ceux qu'ils «bouffent». Mandara et Bornouans se rendant sur les marchés mettent dans leurs poches des fleurs, toujours ouvertes jamais fermées, de Calotropis voire son gui, pour favoriser les négociations. On frappe avec une branche de Calotropis mouillée le bétail que l'on va vendre pour qu'il flatte le regard des acheteurs. Des résidus d'utilisation de charmes protecteurs peuvent se retrouver jusque chez les Arabes Showa. On dispose par exemple une branche de Calotropis devant la maison d'une femme qui vient d'accoucher (P. Jullien de Pommerol 1999 : 1227) moins pour signaler l'événement que pour protéger le nouveau-né de quelques djinn potentiellement menaçants.

10. Nom de nature idéophonique pour évoquer les gros follicules creux de ses fruits, banbambe est le pluriel de wanbambi et la feuille wanbamho. C'est sous la forme du pluriel que Calotropis est le plus souvent désigné. Tous les termes utilisés dans cette troisième partie seront en fulfulde, la langue peule.

11. Rappelons que lekki en fulfulde veut également dire " arbre » et « arbuste ».

12. Le latex de Calotropis est un substitut de celui d'euphorbe non comme principe actif mais comme excipient et conservateur de poison sagittaire de Strophantus sarmentosus dont on enduit les barbules sous la pointe de la flèche.

13. Les musulmans reprennent souvent par analogie des pratiques thérapeutico-magiques païennes. La cendre du feu de l'école coranique, là où le soir on lave les planchettes, servait à frotter le corps des malades touchés par la varicelle (E. Guitard $2014: 239$ ).

14. E. Guitard (2014: 527) relate une semblable recette à Garoua afin d'acquérir de la richesse auprès de Calotropis, "la maison des Jinn ». On découpe une lanière de viande de la hauteur de l'arbuste choisi. À minuit on l'enroule autour du tronc, puis on vient la récupérer, toujours la nuit, pour ensuite la consommer. 


\section{RÉSUMÉS}

Venu des marges du désert Calotropis procera descend progressivement vers le sud, conséquence tout à la fois d'une diffusion anthropique et d'une dégradation du milieu. Crédité de pouvoirs qu'il tirerait de son étrangeté, rarement arbuste n'a autant été sollicité par l'homme. Dans le nord du Cameroun il sert une infinité de rituels de protection, d'ordalies... et fournit le long registre de la pharmacopée.

Coming initially from the desert's margins, Calotropis procera is now progressively moving down south, owing both to anthropic diffusion and to the deterioration of the environment. Calotropis procera is credited with powers which would allegedly stem from its oddity and, as such, it is perhaps one of the most solicited plants in human history. In North Cameroon, it is used for a large number of protection rituals, trials by ordeal... and it also features prominently in traditional medicine's recipes.

\section{INDEX}

Index géographique : Cameroun (nord), Tchad

Mots-clés : Calotropis procera, sécheresse, rituel, ordalie, Giziga Bwi Marva

Keywords : Calotropis procera, rituals and trials by ordeal, Chad, Northern Cameroon, Giziga Bwi Marva

\section{AUTEUR}

\section{CHRISTIAN SEIGNOBOS}

Géographe. Directeur de recherche émérite IRD 